\section{Acesso aos serviços de atenção primária à saúde por adolescentes e jovens em um município do Estado da Bahia, Brasil}

\author{
Adolescent and youth access to primary health \\ care services in a city in the state of Bahia, Brazil
}

\section{Acceso de adolescentes y jóvenes a los servicios de atención primaria en salud en un municipio del Estado de Bahía, Brasil}

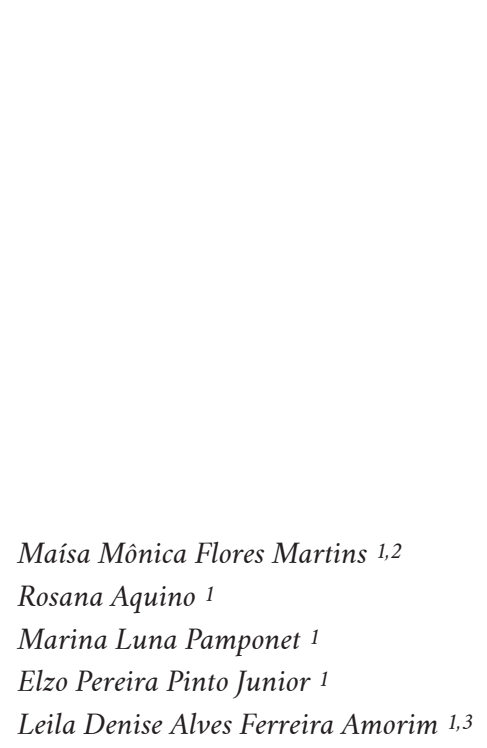

doi: 10.1590/0102-311X00044718

\section{Resumo}

O estudo teve como objetivo analisar a associação entre o acesso aos serviços de atenção primária à saúde dos adolescentes e adultos jovens e a cobertura da Estratégia Saúde da Família (ESF). Trata-se de estudo transversal, a partir de um inquérito domiciliar realizado com 812 individuos de 15 a 24 anos de idade, em Camaçari, Bahia, Brasil, por meio de uma amostragem por conglomerados. Variáveis demográficas, socioeconômicas, de saúde e dos serviços de saúde foram utilizadas para descrição da população, segundo a área de cobertura de atenção primária à saúde. A associação entre área de cobertura da atenção primária à saúde e acesso aos serviços desta, as barreiras de acesso e a participação em atividades educativas foram estimadas por meio da razão de prevalência $(R P)$, com uso do modelo de regressão logística multinível no software $R$. $O$ acesso aos serviços de atenção primária à saúde foi referido por $89,5 \%$ dos indivíduos, não havendo diferenças estatisticamente significantes entre esse tipo de acesso e a área de cobertura da ESF. Entretanto, entre indivíduos residentes em áreas cobertas pela ESF, verificou-se maior acesso às ações de prevenção de agravos e promoção da saúde $(R P=3,0$; IC95\%: 1,68-5,34), mas também menor probabilidade de o atendimento ocorrer no mesmo dia de marcação da consulta $(R P=0,60$; IC95\%: 0,48-0,74) e menor disponibilidade de transporte coletivo ( $R P=0,59$; IC95\%: 0,39-0,90). Os dados deste estudo apontaram elevada prevalência de acesso aos serviços de atenção primátria à saúde entre os adolescentes e adultos jovens. Ainda que não se tenham observado diferenças do acesso entre as áreas com cobertura da ESF, a maior participação desses indivíduos em atividades de prevenção e promoção da saúde reforçam a importância dessa estratégia no âmbito do Sistema Único de Saúde (SUS).

Acesso aos Serviços de Saúde; Atenção Primária à Saúde; Estratégia Saúde da Família; Adolescente
Correspondência

M. M. F. Martins

Instituto de Saúde Coletiva, Universidade Federal da Bahia. Rua Basílio da Gama 316, Salvador, BA 40110-040, Brasil. maisamonica@gmail.com

${ }^{1}$ Instituto de Saúde Coletiva, Universidade Federal da Bahia, Salvador, Brasil.

2 Universidade Católica do Salvador, Salvador, Brasil.

3 Instituto de Matemática e Estatística, Universidade Federal da Bahia, Salvador, Brasil. 


\section{Introdução}

A adolescência é uma fase em que o indivíduo passa a vivenciar condutas de riscos que podem comprometer sua saúde de maneira irreversível, exigindo atenção dos profissionais de saúde, uma vez que há necessidade de reconhecer as demandas dessa população e intervir oportunamente 1. Para satisfazer essas necessidades, é preciso que o adolescente acesse os serviços de saúde. Estudos sobre o acesso aos serviços de saúde podem contribuir para o reconhecimento das desigualdades e das barreiras da organização, além de permitir a identificação de grupos prioritários para atenção à saúde 2,3.

Por muito tempo, adolescentes e adultos jovens foram considerados um grupo populacional saudável, com menor risco de adoecimento e morte 4 . Entretanto, o aumento dos índices de morbimortalidade, devido à maior ocorrência de causas externas 5 , complicações relacionadas à gravidez, doenças sexualmente transmissíveis e fatores de risco para doenças crônicas não transmissíveis nessa faixa etária 6 , demanda iniciativas políticas, sociais, mudanças organizacionais e de práticas dos profissionais dos serviços de saúde 7 . A atenção à saúde do adolescente exige uma abordagem mais criteriosa de cuidados, especialmente no âmbito dos serviços de atenção primária à saúde 8,9.

$\mathrm{O}$ acesso apresenta-se como um dos elementos do sistema de saúde, condicionado por características organizacionais e geográficas, que podem facilitar ou dificultar a entrada dos indivíduos nos serviços de saúde. Abrange características dos indivíduos e dos serviços, que podem viabilizar ou não a utilização dos serviços e a continuidade do cuidado 10,11,12. A atenção primária à saúde, por se constituir como porta de entrada preferencial do sistema de saúde, deve apresentar alguns requisitos específicos que garantam aos usuários maior facilidade de acesso, como menor distância do domicílio aos serviços, maior flexibilidade na marcação de consultas e nos horários de funcionamento, dentre outros 13 .

No Brasil, a Pesquisa Nacional de Saúde do Escolar verificou que 48\% dos entrevistados procuraram por algum serviço ou profissional de saúde nos últimos 12 meses anteriores à entrevista, sendo a unidade básica de saúde (UBS) o serviço mais referido (47,5\%). Entre aqueles que procuraram a UBS, $85,1 \%$ afirmaram ter obtido atendimento 14 .

A disposição geográfica dispersa 9 , a falta de informação sobre os meios de obtenção do cuidado e a ausência de transporte para chegar aos serviços de saúde ${ }^{15}$, além de fatores relacionados a características organizacionais 16 , têm sido referidos como barreiras de acesso aos serviços de saúde, tanto em estudos com população adulta quanto com adolescentes. Outras barreiras relatadas especificamente por adolescentes incluem a preocupação sobre a confidencialidade, o desconhecimento dos serviços e o desconforto em compartilhar suas preocupações sobre a saúde com os profissionais 16 .

Alguns estudos ressaltaram que adolescentes e adultos jovens mantêm certo distanciamento dos serviços de saúde, especialmente daqueles que prestam cuidados primários. Por outro lado, as equipes de saúde também apresentam dificuldades para acolhê-los 16,17,18. Outros autores destacaram que os adolescentes pouco utilizam os serviços para prevenção e promoção da saúde ${ }^{19}$, restringindo a procura a ações curativas e individuais 20 .

Estudos sobre acesso aos serviços de atenção primária à saúde entre adolescentes e jovens são pouco frequentes no Brasil, sendo a maioria específicos para um determinado tipo de cuidado, como gravidez na adolescência, saúde bucal e saúde mental 19,21,22. Essa carência de estudos aponta a necessidade de investigações nesse âmbito, a fim de contribuir com achados que subsidiem o redirecionamento das práticas assistenciais dos serviços de atenção primária à saúde 23 , assim como para o planejamento de ações e políticas para esse ciclo de vida. O presente estudo tem por objetivo analisar a associação entre o acesso aos serviços de atenção primária à saúde por parte dos adolescentes e adultos jovens e a cobertura da Estratégia Saúde da Família (ESF) em um município do Estado da Bahia, Brasil. Adicionalmente, foram investigados possíveis mecanismos facilitadores ou dificultadores do acesso, de forma a compreender a associação estudada. 


\section{Métodos}

Foi estruturado um modelo teórico com a perspectiva de nortear as abordagens metodológicas do estudo. Esse modelo propõe que o fato de residir em áreas cobertas pela ESF está associado ao acesso aos serviços de atenção primária à saúde, devido às suas características organizacionais e geográficas, que podem reduzir as barreiras que dificultam a entrada dos usuários a esses serviços. Aspectos individuais e contextuais, que também influenciam no acesso aos serviços, foram considerados, como as características demográficas, socioeconômicas, individuais, de saúde e das famílias. Finalmente, destaca-se que, para investigação desta proposição, é importante ainda considerar as necessidades dos indivíduos que podem representar a demanda por serviços de saúde, podendo ser influenciadas por outras condições que determinam a decisão dos indivíduos em buscar ou não um determinado serviço (Figura 1).

\section{Desenho do estudo}

Estudo transversal, a partir de um inquérito domiciliar realizado com indivíduos de 15-24 anos de idade, desenvolvido no Município de Camaçari, no período de outubro de 2011 a janeiro de 2012. O estudo faz parte do projeto Avaliação dos Hábitos de Vida e Acessibilidade aos Serviços de APS da População entre 15 e 24 Anos 24.

Em 2010, o Município de Camaçari, localizado na Região Metropolitana de Salvador, apresentava população de 242.970 habitantes, com aproximadamente $96 \%$ dos residentes concentrados na área urbana (Instituto Brasileiro de Geografia e Estatística. https://cidades.ibge.gov.br/brasil/ba/camaca ri/panorama, acessado em 12/Dez/2015). Em 2011, a rede de saúde era composta por 38 unidades, sendo 28 da ESF, cuja cobertura estimada era de 49,7\% 25. Segundo informações do Sistema de Informação da Atenção Básica, foi possível identificar 43.544 famílias cadastradas por agentes comunitários

\section{Figura 1}

Modelo teórico para avaliação da associação entre o acesso aos serviços de saúde por adolescentes e adultos jovens e a área de cobertura da atenção primária à saúde. Camaçari, Bahia, Brasil, 2012.

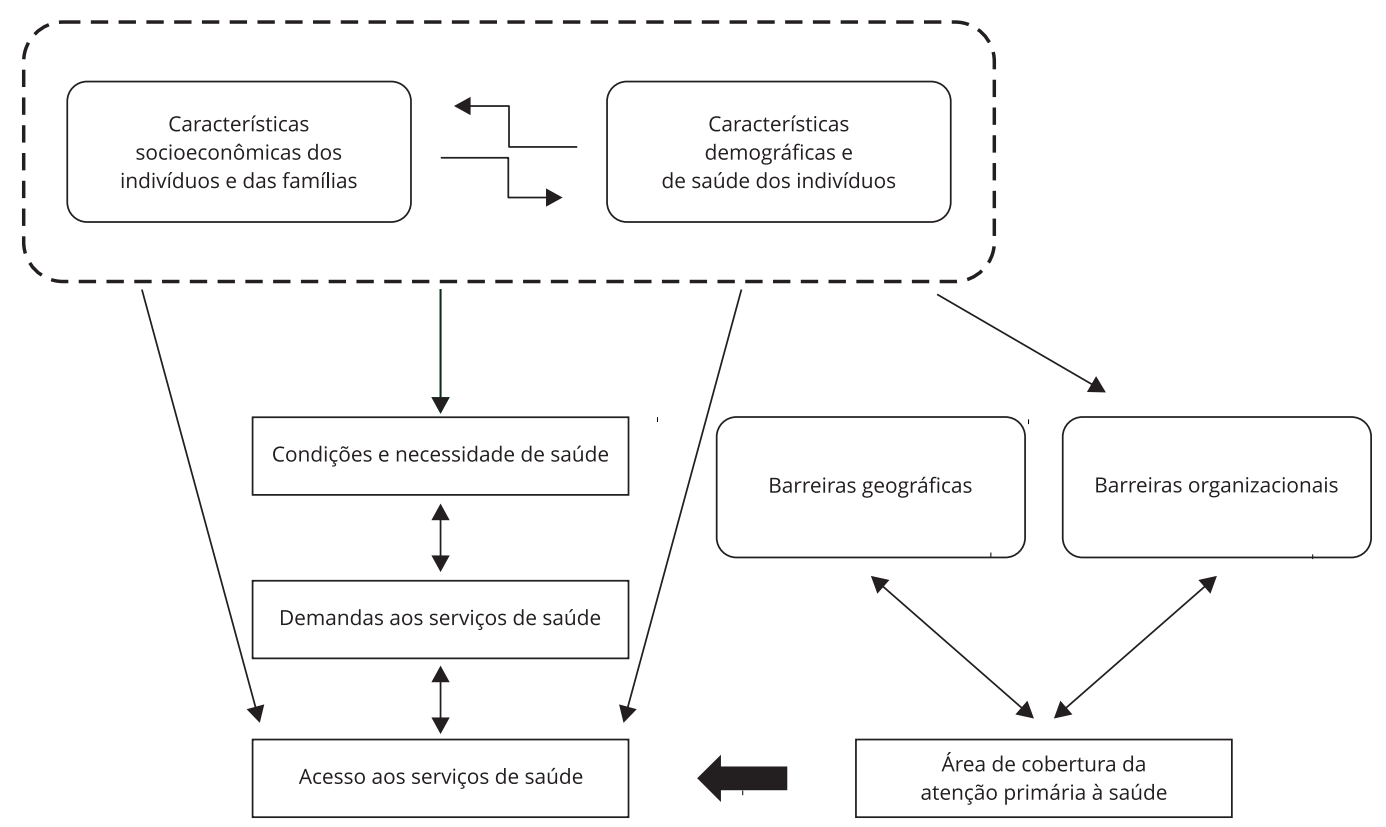


de saúde (ACS), sendo a proporção de cobertura populacional estimada em $58 \%$, em dezembro de 2011 (Departamento de Informática do SUS. http://tabnet.datasus.gov.br/cgi/deftohtm.exe?siab/ cnv/SIABFba.def, acessado em 15/Set/2016).

O conjunto de dados do inquérito foi obtido por meio de uma amostragem probabilística por conglomerados em dois estágios de seleção: unidade primária - microárea; unidade secundária - domicílio. Para o primeiro estágio da seleção, utilizou-se uma listagem da Secretaria Municipal de Saúde com informações sobre todas as 477 microáreas. Dessas microáreas, 63 foram sorteadas e incluídas no estudo, sendo 30 unidades de ESF e 33 UBS. Do total de microáreas, apenas três não tinham cobertura de ACS. Para a construção do segundo estágio de seleção da amostragem, inicialmente foi realizado o sorteio dos logradouros e, em seguida, em cada logradouro sorteado, foi identificado o domicílio de menor número, sendo este o primeiro a ser visitado para a coleta dos dados. $\mathrm{O}$ entrevistador seguia domicílio a domicílio em um mesmo lado da rua e, ao final do trecho, modificava-se o trajeto para o outro lado do logradouro (se pertencesse à microárea).

Ao final, o entrevistador seguia para o próximo logradouro sorteado na microárea até completar a amostra prevista de domicílios. Após identificação dos domicílios com pelo menos um residente entre 15 e 24 anos, realizava-se um sorteio aleatório, quando necessário, de um morador por domicílio, nessa faixa etária, para responder ao questionário, mesmo em casos em que o indivíduo não estivesse presente no momento. Ressalta-se que gestantes, pessoas com necessidades especiais (que inviabilizassem a aplicação do questionário) e empregados domésticos daqueles domicílios identificados dentro da faixa etária foram excluídos do sorteio.

Informações mais detalhadas do cálculo do tamanho da amostra e da composição da amostragem podem ser obtidas em Aquino 24, mas vale destacar que a amostra foi calculada utilizando um estimativas associadas a desfecho de menor prevalência, além de considerar o tamanho dos conglomerados. Portanto, acredita-se que nossa abordagem tenha poder suficiente para detectar as diferenças dessa análise. Em resumo, o tamanho amostral foi estimado em 1.800 adolescentes e adultos jovens (15-24 anos), com o objetivo de avaliar os efeitos da ESF na adoção de hábitos saudáveis e na acessibilidade aos serviços de atenção primária à saude na população do Município de Camaçari, em 2011, sendo a amostra final de 1.701 indivíduos.

Para as análises do presente estudo, foram considerados os indivíduos que referiram ter procurado atendimento de saúde na unidade de referência do seu domicílio nos últimos 12 meses anteriores à entrevista. Desse modo, neste trabalho são considerados dados de 812 adolescentes, que representam $47,5 \%$ da amostra total.

\section{Variáveis estudadas}

A principal variável dependente do estudo foi o acesso aos serviços de atenção primária à saúde (sim ou não), sendo verificado por meio das respostas afirmativas para as seguintes perguntas: "Procurou atendimento na unidade de referência do seu domicilio nos últimos 12 meses?" e "Conseguiu ser atendido?". Além disso, como uma proxy de acesso a ações de prevenção e promoção da saúde (sim ou não), foi analisada uma variável dependente secundária, a partir do seguinte questionamento: "Participou de alguma atividade coletiva realizada por profissionais da saúde na Unidade de referência nos últimos 12 meses?”.

A variável independente principal foi definida como o tipo de unidade de atenção primária à saúde de referência da área do domicílio do entrevistado, sendo consideradas áreas cobertas pela ESF e áreas não cobertas pela ESF. É importante destacar que as áreas não cobertas pela ESF foram compostas de UBS tradicionais e UBS localizadas em estabelecimentos que integravam Unidade de Pronto Atendimento (UPA).

Para investigar possíveis mecanismos facilitadores ou dificultadores do acesso, foram analisadas as seguintes variáveis: (1) tempo entre a marcação da consulta e o atendimento (atendido no mesmo dia ou em dois e mais dias); (2) tempo de deslocamento a pé do domicílio à unidade de saúde de referência ( $\leq 15$ minutos ou $>15$ minutos), cuja estratificação foi baseada em estudos que apontaram 15 minutos como um tempo razoável para o indivíduo percorrer a distância entre sua residência e o serviço de saúde a pé 26,27; (3) disponibilidade de transporte coletivo (sim ou não); (4) filas para marcação de consultas (sim ou não); e (5) dias específicos para a marcação de consultas (sim ou não). 
As outras covariáveis são apresentadas de acordo os seguintes grupos de características: (1) características demográficas dos indivíduos: sexo (masculino ou feminino), faixa etária (15-19 anos ou 20-24 anos), cor da pele (branco ou preto, pardo, amarelo e indígena), escolaridade (até Ensino Médio/ técnico incompleto, ou Ensino Médio/técnico completo a Ensino Superior), situação conjugal (solteiro ou casado/divorciado/separado/viúvo), filhos (sim ou não); (2) características socioeconômicas dos indivíduos e das famílias: trabalho (sim ou não), trabalho do responsável (sim ou não), auxílio financeiro - Bolsa Família (sim ou não), escolaridade do responsável pela família (analfabeto até Ensino Fundamental completo ou Ensino Médio incompleto até Ensino Superior), e plano de saúde (plano médico e odontológico ou não); (3) condições de saúde dos indivíduos e características dos serviços de saúde: percepção da condição de saúde (muito boa/boa ou regular/ruim), presença de alguma doença ou problema de saúde (sim ou não), motivo da procura pelo atendimento nos serviços de saúde de referência (doença/agravo ou ações preventivas) e conhecer o agente comunitário de saúde ACS que visita seu domicílio (sim ou não).

\section{Análise estatística}

Para verificar se as áreas com e sem cobertura ESF diferiam de acordo com as demais covariáveis, realizou-se uma análise descritiva da distribuição dessas covariáveis, segundo a área de cobertura da atenção primária à saúde (variável independente principal), com apresentação das correspondentes frequências absolutas e relativas. Para essas análises bivariadas, usou-se o teste qui-quadrado de Pearson, com correção de Rao-Scott de segunda ordem para o desenho amostral 28,29, considerando a estrutura de conglomerados dos dados. Essa análise foi realizada com base no comando svy: tabulate do software Stata 12.0 (https://www.stata.com). Para todas as análises, considerou-se um nível de 5\% de significância.

Estimou-se, por meio do modelo nulo da regressão logística multinível, o coeficiente de correlação intraclasse (ICC) para a variável resposta principal (acesso aos serviços de atenção primária à saúde), que foi de 0,10. Sabe-se que, em situações em que os eventos são muito frequentes, a odds ratio $(\mathrm{OR})$ não fornece uma boa estimativa da razão de prevalência (RP), pois geralmente superestima a magnitude dessa medida de associação ${ }^{30}$. Desse modo, são apresentadas estimativas pontuais para as RP e seus respectivos intervalos de 95\% de confiança (IC95\%). A RP foi estimada pelo modelo de regressão logística multinível, considerando-se o método delta, com padronização condicional ${ }^{29}$, por meio do uso do pacote prLogistic no software R (https://rdrr.io/cran/prLogistic/). O método delta é uma técnica utilizada para calcular o erro padrão em amostragens complexas 31 , ao passo que a padronização condicional é um dos métodos usados para estimação de RP com o modelo logístico, em que as medidas de efeitos são calculadas para valores de referência das covariáveis, como exemplo, a média das covariáveis quantitativas 30 . As estimativas da RP foram obtidas dentre aquelas definidas no modelo teórico, ajustando-se pelas covariáveis selecionadas após avaliação de colinearidade. Com exclusão das covariáveis potencialmente correlacionadas com as que permaneceram no modelo, a avaliação de colinearidade foi feita por meio do uso do teste qui-quadrado, com correção de Rao-Scott 28,29. Assim, no modelo final, as seguintes covariáveis foram incluídas: área de cobertura da atenção primária à saúde (variável independente principal), sexo, faixa etária, escolaridade, trabalho e percepção de saúde.

Com o propósito de realizar análise de sensibilidade para verificar a robustez das estimativas de RP com os modelos logísticos multiníveis, utilizou-se também o modelo Poisson multinível para estimar RP, e estimou-se ainda a OR por meio de modelos logísticos multiníveis. Apesar do uso frequente do modelo Poisson em literatura recente para estimação de RP, essa metodologia produz, de modo geral, estimativas menos precisas (intervalos de confiança mais amplos) por aproximar resultados para respostas binárias, uma vez que a distribuição Poisson é matematicamente definida para análise de respostas discretas (contagens), aproximando, em algumas situações, respostas binomiais (ou binárias, como caso particular) 31. Todas as análises foram realizadas no software Stata 12.0.

A pesquisa de origem foi submetida e aprovada pelo Comitê de Ética em Pesquisa do Instituto de Saúde Coletiva, Universidade Federal da Bahia, por meio do parecer de oo 019/2009. 


\section{Resultados}

Do total de 812 adolescentes e adultos jovens que referiram ter procurado o serviço de atenção primária à saúde de referência do seu domicílio, 89,5\% $(\mathrm{n}=727)$ tiveram acesso nos últimos 12 meses. O relato de acesso entre aqueles que residiam em áreas cobertas pela ESF foi de 87,3\%, ao passo que entre os que residiam em áreas não cobertas pelas ESF foi de 91,6\% (Tabela 1). Dentre os motivos relatados pelos participantes da não procura pelos serviços de referência de seu domicílio nos últimos 12 meses, destacam-se o fato de não terem precisado $(58,7 \%)$, possuírem plano de saúde $(15,1 \%)$, terem dificuldade de encontrar atendimento (7,7\%), não gostarem da qualidade do atendimento (4,5\%), além da falta de oferta do serviço de que necessitavam (3,3\%), a distância do serviço de saúde $(1,4 \%)$, o horário incompatível (1,2\%), entre outros motivos $(8,1 \%)$.

Em relação às características demográficas dos entrevistados, pode-se observar uma maior proporção de indivíduos do sexo feminino (67,4\%), de 15-19 anos $(55,7 \%)$ e cor da pele autorreferida preta, parda, amarela ou indígena (90,9\%). Sobre as características socioeconômicas, $67,1 \%$ não trabalhavam e 70,6\% dos seus responsáveis apresentavam vínculos empregatícios. Além disso, a maioria dos adolescentes e adultos jovens (58,5\%) considerava sua saúde muito boa ou boa, e não referia apresentar doença $(78,9 \%)$ no momento da entrevista. Os grupos residentes em áreas cobertas e não cobertas pela ESF apresentavam características semelhantes para todas essas variáveis (Tabela 1).

Na comparação das características dos residentes em áreas cobertas e não cobertas pela ESF, foram observadas diferenças estatisticamente significante nas variáveis escolaridade (valor de $\mathrm{p}=0,05$ ), situação conjugal (valor de $\mathrm{p}=0,02$ ), filhos (valor de $\mathrm{p}=0,00$ ), auxílio Bolsa Família (valor de $\mathrm{p}=0,00$ ), plano privado de saúde (valor de $\mathrm{p}=0,02$ ), motivo de procurar atendimento em unidade de referência (valor de $\mathrm{p}=0,00$ ) e ACS conhecido (valor de $\mathrm{p}=0,00$ ). Essas diferenças indicavam que os residentes em área cobertas pela ESF apresentavam maiores percentuais de indivíduos com grau de escolaridade até Ensino Médio/técnico incompleto, casados/divorciados/separados/viúvos, com filhos e beneficiários do auxílio Bolsa Família, além de menores percentuais de cobertura de plano privado de saúde. Ainda em relação aos residentes em áreas cobertas pela ESF, foi maior o percentual dos que procuram pelos serviços para ações preventivas e o percentual de entrevistados que conheciam o ACS (Tabela 1).

$\mathrm{Na}$ análise com modelo de regressão logística multinível, observou-se que não houve associação estatisticamente significante entre o acesso aos serviços de atenção primária à saúde e a cobertura da ESF. Dentre as variáveis de ajuste, apenas sexo apresentou diferença estatisticamente significante, sendo que as adolescentes tiveram um acesso $5 \%$ menor aos serviços de saúde de áreas cobertas pela ESF (RP = 0,95; IC95\%: 0,91-0,99) do que os adolescentes do sexo masculino (Tabela 2). Nota-se, ainda, que as estimativas obtidas pelo modelo Poisson multinível não apresentaram associação estatisticamente significante ( $\mathrm{RP}=0,96$; IC95\%: 0,82-1,11), provavelmente por apresentarem intervalos de confiança mais amplos. Destaca-se também que, quando analisado por meio do modelo logístico multinível (OR = 0,61; IC95\%: 0,34-1,09), verifica-se que a OR superestima a RP para a maioria das covariáveis (Tabela 2).

A participação em atividades educativas (proxy de acesso a ações de prevenção e promoção da saúde) foi três vezes maior entre os residentes de áreas cobertas pela ESF (RP = 3,00; IC95\%: 1,68-5,34) (Tabela 3).

Quanto às barreiras de acesso aos serviços de saúde, entre os residentes de áreas cobertas por ESF, foi menor a referência a atendimento no mesmo dia da marcação da consulta ( $R P=0,60$; IC95\%: 0,48-0,74) e presença de transporte coletivo ( $R P=0,59$; IC95\%: 0,39-0,90). Não houve diferença estatisticamente significante para tempo de deslocamento para unidade e existência de filas e de dias específicos para marcação de consultas segundo a presença de ESF na área de residência dos adolescentes e adultos jovens (Figura 2). 
Tabela 1

Características demográficas, socioeconômicas e das condições de saúde de adolescentes, adultos jovens e familiares, de acordo com a área de cobertura dos serviços da atenção primária à saúde. Camaçari, Bahia, Brasil, 2012.

\begin{tabular}{|c|c|c|c|c|c|}
\hline \multirow[t]{2}{*}{ Variáveis } & \multicolumn{5}{|c|}{ Área de cobertura } \\
\hline & $\mathbf{n}$ & $\%$ & $\begin{array}{c}\text { Área coberta pela ESF } \\
\%\end{array}$ & $\begin{array}{c}\text { Área não coberta pela ESF } \\
\%\end{array}$ & Valor de $p$ * \\
\hline Total & 812 & 100,0 & 48,4 & 51,6 & \\
\hline Acesso & & & & & 0,11 \\
\hline Sim & 727 & 89,5 & 87,3 & 91,6 & \\
\hline Não & 85 & 10,5 & 12,7 & 8,4 & \\
\hline Sexo & & & & & 0,97 \\
\hline Masculino & 265 & 32,6 & 32,6 & 32,7 & \\
\hline Feminino & 547 & 67,4 & 67,4 & 67,3 & \\
\hline Faixa etária (anos) & & & & & 0,92 \\
\hline $15-19$ & 452 & 55,7 & 55,5 & 55,8 & \\
\hline $20-24$ & 360 & 44,3 & 44,5 & 44,2 & \\
\hline Cor da pele & & & & & 0,38 \\
\hline Branca & 74 & 9,1 & 8,1 & 10 & \\
\hline Preta, parda, amarela e indígena & 738 & 90,9 & 91,9 & 90 & \\
\hline Escolaridade & & & & & 0,05 \\
\hline Até Ensino Médio/Técnico incompleto & 530 & 66,1 & 69,8 & 62,6 & \\
\hline Ensino Médio/Técnico completo a Ensino Superior & 272 & 33,9 & 30,2 & 37,4 & \\
\hline Situação conjugal & & & & & 0,02 \\
\hline Solteiro & 573 & 70,6 & 65,1 & 75,7 & \\
\hline Casado/Divorciado/Separado/Viúvo & 239 & 29,4 & 34,9 & 24,3 & \\
\hline Filhos & & & & & 0,00 \\
\hline $\operatorname{Sim}$ & 215 & 26,5 & 32,8 & 20,5 & \\
\hline Não & 597 & 73,5 & 67,2 & 79,5 & \\
\hline Trabalho & & & & & 0,18 \\
\hline Sim & 267 & 32,9 & 30,5 & 35,1 & \\
\hline Não & 545 & 67,1 & 69,5 & 64,9 & \\
\hline Trabalho do responsável & & & & & 0,88 \\
\hline Sim & 568 & 70,6 & 70,9 & 70,3 & \\
\hline Não & 237 & 29,4 & 29,1 & 29,7 & \\
\hline Auxílio do Bolsa Família & & & & & 0,00 \\
\hline Sim & 316 & 39,2 & 45,3 & 33,4 & \\
\hline Não & 491 & 60,8 & 54,7 & 66,6 & \\
\hline Plano privado de saúde & & & & & 0,02 \\
\hline Médico e odontológico ou odontológico & 132 & 17,6 & 13,5 & 21,6 & \\
\hline Não & 616 & 82,4 & 86,5 & 78,4 & \\
\hline Escolaridade do responsável & & & & & 0,39 \\
\hline Analfabeto até Ensino Fundamental completo & 423 & 56,9 & 59,1 & 54,9 & \\
\hline Ensino Médio incompleto até Ensino Superior & 320 & 43,1 & 40,9 & 45,1 & \\
\hline Percepção de saúde & & & & & 0,99 \\
\hline Muito boa/Boa & 475 & 58,5 & 58,5 & 58,5 & \\
\hline Regular/Ruim & 337 & 41,5 & 41,5 & 41,5 & \\
\hline Refere apresentar doença & & & & & 0,66 \\
\hline Sim & 171 & 21,1 & 21,6 & 20,5 & \\
\hline Não & 641 & 78,9 & 78,4 & 79,5 & \\
\hline $\begin{array}{l}\text { Motivo de procurar atendimento em unidade de } \\
\text { referência }\end{array}$ & & & & & 0,00 \\
\hline Doença/Agravo & 404 & 49,7 & 39,9 & 58,9 & \\
\hline Ações preventivas & 408 & 50,3 & 60,1 & 41,1 & \\
\hline Conhece o ACS & & & & & 0,00 \\
\hline Sim & 623 & 76,7 & 38,2 & 9,3 & \\
\hline Não & 189 & 23,3 & 61,8 & 90,7 & \\
\hline
\end{tabular}

ACS: agente comunitário de saúde; ESF: Estratégia Saúde da Família. 
Tabela 2

Estimativas usando modelos multiníveis para a associação entre a área de cobertura da atenção primária à saúde e o acesso aos serviços de saúde entre adolescentes e adultos jovens, ajustados por covariáveis, Camaçari, Bahia, Brasil, 2012.

\begin{tabular}{|c|c|c|c|c|c|c|}
\hline \multirow[t]{2}{*}{ Variáveis } & \multicolumn{2}{|c|}{$\begin{array}{c}\text { Modelo logístico } \\
\text { multinível }\end{array}$} & \multicolumn{2}{|c|}{$\begin{array}{c}\text { Modelo Poisson } \\
\text { multinível }\end{array}$} & \multicolumn{2}{|c|}{$\begin{array}{c}\text { Modelo logístico } \\
\text { multinível }\end{array}$} \\
\hline & $\mathbf{R P}$ & IC95\% & $\mathbf{R P}$ & IC95\% & OR & IC95\% \\
\hline \multicolumn{7}{|l|}{ Área de cobertura } \\
\hline Área coberta pela ESF & 0,97 & $0,93-1,01$ & 0,96 & $0,82-1,11$ & 0,61 & $0,34-1,09$ \\
\hline \multicolumn{7}{|l|}{ Sexo } \\
\hline Feminino & 0,95 & $0,91-0,99$ & 0,94 & $0,80-1,10$ & 0,47 & $0,26-0,87$ \\
\hline \multicolumn{7}{|l|}{ Faixa etária (anos) } \\
\hline $20-24$ anos & 0,97 & $0,94-1,01$ & 0,96 & $0,81-1,13$ & 0,64 & $0,38-1,08$ \\
\hline \multicolumn{7}{|l|}{ Escolaridade } \\
\hline Ensino Médio/Técnico completo a Ensino Superior & 1,01 & $0,98-1,03$ & 1,02 & $0,86-1,20$ & 1,17 & $0,68-2,00$ \\
\hline \multicolumn{7}{|l|}{ Trabalho } \\
\hline Sim & 0,99 & $0,97-1,02$ & 0,99 & $0,84-1,18$ & 0,99 & $0,57-1,70$ \\
\hline \multicolumn{7}{|l|}{ Percepção de saúde } \\
\hline Muito boa/Boa & 1,02 & $0,99-1,04$ & 0,94 & $0,77-1,14$ & 1,55 & $0,96-2,49$ \\
\hline
\end{tabular}

ESF: Estratégia Saúde da Família; IC95\%: intervalo de 95\% de confiança; OR: odds ratio; RP: razão de prevalência.

Tabela 3

Razão de prevalência (RP) ajustada da associação entre presença de Estratégia Saúde da Família (ESF) na área de residência e participação em atividades educativas entre adolescentes e adultos jovens. Camaçari, Bahia, Brasil, 2012.

\begin{tabular}{|c|c|c|}
\hline \multirow[t]{2}{*}{ Variáveis } & \multicolumn{2}{|c|}{ Modelo logístico multinível } \\
\hline & $\mathbf{R P}$ & $\mathrm{IC} 95 \%$ \\
\hline \multicolumn{3}{|l|}{ Área de cobertura } \\
\hline Área coberta pela ESF & 3,00 & $1,68-5,34$ \\
\hline \multicolumn{3}{|l|}{ Sexo } \\
\hline Feminino & 0,94 & $0,52-1,69$ \\
\hline \multicolumn{3}{|l|}{ Faixa etária (anos) } \\
\hline $20-24$ & 1,35 & $0,78-2,32$ \\
\hline \multicolumn{3}{|l|}{ Trabalho } \\
\hline Sim & 0,61 & $0,33-1,15$ \\
\hline \multicolumn{3}{|l|}{ Escolaridade } \\
\hline Ensino Médio/Técnico completo a Ensino Superior & 1,38 & $0,79-2,40$ \\
\hline \multicolumn{3}{|l|}{ Percepção de saúde } \\
\hline Muito boa/Boa & 0,87 & $0,52-1,46$ \\
\hline
\end{tabular}

IC95\%: intervalo de 95\% de confiança.

\section{Discussão}

Os dados deste estudo apontaram que quase 90\% dos adolescentes e adultos jovens obtiveram acesso aos serviços de atenção primária à saúde de referência do seu domicílio nos últimos 12 meses, não havendo diferença estatisticamente significante entre os residentes de áreas cobertas e não cobertas pela ESF. Entretanto, diferenças marcantes foram observadas com respeito ao acesso às ações de prevenção de agravos e promoção da saúde, sendo três vezes maior a participação em atividades 


\section{Figura 2}

MRazão de prevalência (RP) e respectivos intervalos de 95\% de confiança (IC95\%), segundo modelo logístico multinível, para avaliar o efeito da presença de Estratégia Saúde da Família (ESF) na área de residência do adolescente e jovem adulto, nas características de acessibilidade geográfica e organizacional dos serviços de saúde. Camaçari, Bahia, Brasil, 2012.

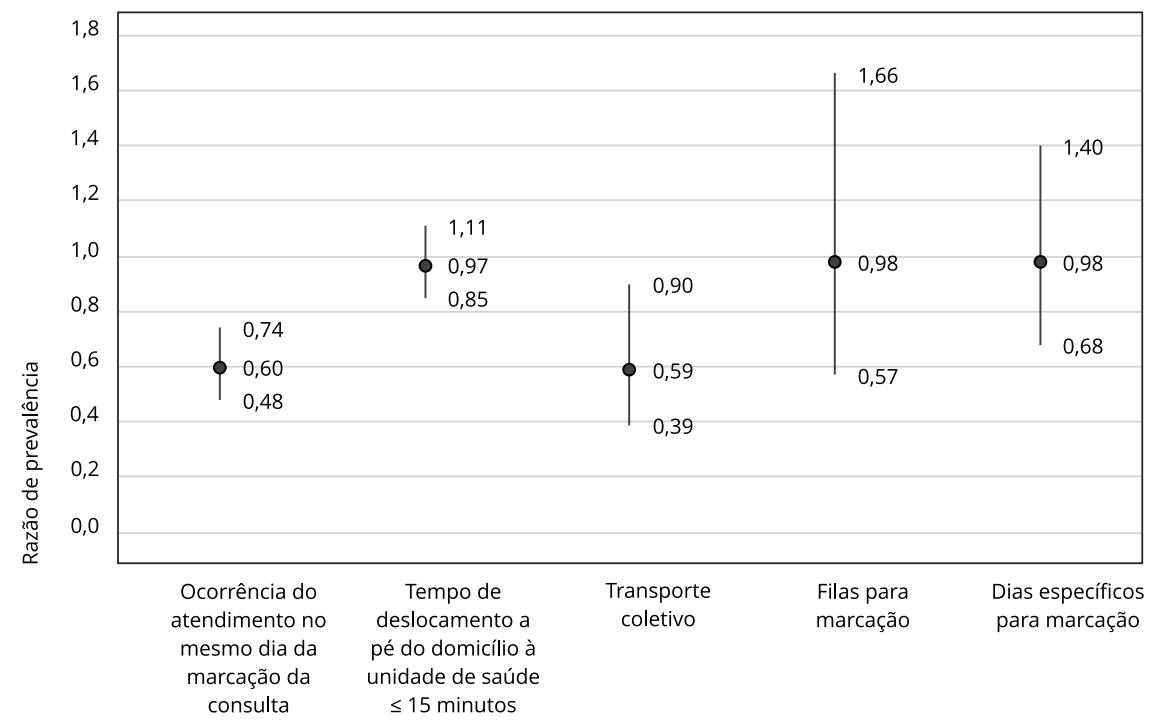

educativas nos serviços da ESF. Também houve maiores percentuais de indivíduos que procuraram por esses serviços para ações preventivas e que conheciam o ACS nas áreas cobertas pela ESF.

O amplo acesso aos serviços primários de saúde é um achado semelhante ao de outros estudos 32,33,34, representando importante avanço na implementação do SUS. Entretanto, uma vez que é direito do cidadão e dever do Estado garantir o acesso aos serviços de saúde, seria esperado que todos os jovens que procuraram atendimento tivessem sido atendidos 35. Mesmo que os percentuais de não acesso sejam baixos, não podem ser negligenciados, pois indicam que os indivíduos que necessitaram de cuidados de saúde não conseguiram entrar no primeiro nível de atenção do sistema de saúde, uma vez que a ESF surge como a conformação de um serviço de porta de entrada preferencial do sistema ${ }^{36}$.

$\mathrm{O}$ fato de não terem sido encontradas diferenças estatisticamente significantes no acesso aos serviços de saúde e às áreas de cobertura da atenção primária à saúde deve ser analisado com base em dois aspectos. Primeiro, as áreas cobertas pela ESF apresentaram indicadores de maior vulnerabilidade social, conformando um cenário geralmente associado a menor acesso aos serviços de saúde. Segundo, entre as unidades de saúde das áreas não cobertas estavam incluídas UBS localizadas em estabelecimentos que integravam UPA. Assim, a não observância de diferenças entre áreas socialmente desiguais e cobertas por unidades com diferentes estruturas organizacionais pode indicar que, em vez de um efeito negativo, a ESF, no município, representou um potencial indutor de equidade, dado que as áreas cobertas por essa modalidade de atenção primária à sáude alcançaram percentuais de acesso aos serviços semelhantes àqueles de áreas socialmente mais favorecidas.

Outro achado que chama atenção nesse estudo foi a menor probabilidade de adolescentes do sexo feminino acessarem serviços de atenção primária à saúde em áreas cobertas pela ESF, quando comparadas com adolescentes do sexo masculino. Esse resultado pode ser explicado pelo fato de que as adolescentes, ao alcançarem a puberdade e com a sua iniciação sexual, procuram com maior frequência os serviços de saúde em busca de atendimento ginecológico, que é feito, na maioria das vezes, por ginecologistas e, portanto, em serviços que contem com essa especialidade médica 37. 
Adicionalmente, as grandes diferenças encontradas quanto aos motivos de procura aos serviços e a participação em atividades educativas demonstraram que, em áreas cobertas pela ESF, os serviços de atenção primária à saúde são identificados e utilizados pelos adolescentes e jovens. Isso indica que as unidades de saúde não atendem apenas as necessidades de atenção aos problemas do setor, mas funcionam como serviços capazes de responder a demandas por prevenção de doenças e promoção da saúde, o que deve ser destacado como fortemente positivo e condizente com as diretrizes e os propósitos da ESF. Embora tenhamos identificado um estudo com resultados semelhantes 4, nossos achados são diferentes da maioria dos encontrados na literatura, que destacam que os adolescentes restringem a sua busca de serviços de saúde a ações de caráter curativista de um problema já instalado $19,20,38,39$.

O cuidado à saúde dos adolescentes e adultos jovens pressupõe a necessidade de horizontalização entre as ações e as relações usuários/profissionais para favorecer o acolhimento, o vínculo e o efetivo acesso ${ }^{40}$. Para o alcance das necessidades de saúde desses jovens, faz-se necessário o envolvimento dos trabalhadores na atenção integral, a fim de dar resolubilidade aos problemas dessa população, abrangendo ações preventivas e curativas.

O estudo evidenciou a existência de barreiras de acesso aos serviços de atenção primária à saúde, as quais impõem restrições ao processo de obtenção do cuidado, impactando na qualidade de vida dos indivíduos, além de contribuir com a redução de sobrevida 41 e dificultar a sua efetivação. $\mathrm{O}$ tempo entre a marcação e a consulta e a ausência de transporte coletivo representaram barreiras de acesso associadas às áreas cobertas pela ESF, contrapondo-se às diretrizes propostas na organização das suas ações e serviços.

Dentre as barreiras geográficas, verificou-se que os adolescentes e adultos jovens que residiam em áreas cobertas pela ESF apresentaram maior probabilidade de gastar mais tempo de deslocamento a pé do domicílio à unidade de saúde e menor disponibilidade de transporte coletivo, quando comparados aos indivíduos que moram em áreas não cobertas pela ESF. Apesar da importância do transporte coletivo no deslocamento em cidades de médio e grande porte, estudos demonstram que o deslocamento a pé é a principal forma de acessibilidade geográfica aos serviços da ESF 42,43. Apesar dessas questões não terem sido investigadas pelo estudo, os indivíduos que residem em áreas cobertas pela ESF apresentam maiores condições de vulnerabilidade social, o que pode repercutir em baixas condições de custeio financeiro de um transporte coletivo para deslocamento até a unidade. Esse fato é destacado pela literatura como uma barreira de um acesso facilitado, entretanto o deslocamento a pé é o mais utilizado pelos usuários de serviços da ESF 43.

Fatores ligados a acessibilidade organizacional, como o não atendimento no mesmo dia da marcação da consulta e a existência de filas, além de dias específicos para esse fim, também foram observados como potenciais dificultadores do acesso. Esses achados corroboram outros estudos ao demonstrar que tempo superior a sete dias de espera entre a marcação e a consulta 38 e que demora para conseguir $\mathrm{o}$ atendimento 40 aumentam as chances de não efetivar o acesso. Adicionalmente, um estudo realizado em um município do interior da Bahia destacou que a restrição da marcação de consulta a dias específicos dificultava o acesso aos serviços da ESF 44. Dentre os dificultadores do acesso, o presente estudo não encontrou diferenças entre as áreas cobertas pela atenção primária à saúde e o desfecho que analisou a existência de filas para marcação de consultas. Vale destacar que estudos que analisaram o tempo na fila de espera verificaram uma maior frequência entre os indivíduos de menor escolaridade 35 . É importante ressaltar que os serviços de saúde acessados por usuários de áreas não cobertas pela ESF representam modalidades distintas de atenção. A existência de duas modalidades distintas na categoria "áreas não cobertas pela ESF" pode ter influenciado as frequências de filas e dias específicos para marcação de consultas, haja vista que nas unidades localizadas com serviços com características de pronto atendimento, essas variáveis apresentariam menores valores quando comparados às UBS tradicionais. Desse modo, ao unir as duas modalidades de atenção na categoria "áreas não cobertas pela ESF", o padrão de respostas se aproxima dos resultados observados na categoria "áreas cobertas pela ESF".

A persistência de problemas relacionados a acessibilidade geográfica e organizacional é fator que pode contribuir para o afastamento dos adolescentes e adultos jovens dos serviços da ESF. Destaca-se que a organização dos serviços deve ser superada, uma vez que, não seriam esperadas melhores condições para os indivíduos que residem em áreas cobertas. 
A autopercepção de saúde tem sido utilizada como um bom indicador na avaliação do comportamento dos indivíduos na busca por serviços de saúde 4,45. O percentual de adolescentes e adultos jovens que avaliou seu estado de saúde como regular ou ruim foi muito superior a outros estudos da literatura 4,8,33. Esses são achados preocupantes para um público que, por muito tempo, foi considerado saudável. Outros fatores que podem apresentar uma forte relação com esses resultados são as questões contextuais, sociais e culturais dos indivíduos que necessitam ser mais bem investigadas.

Algumas limitações precisam ser ponderadas para a interpretação dos achados. Primeiro, por se tratar de um estudo de delineamento transversal, não é possível realizar inferências causais. Segundo, o estabelecimento da variável independente principal pode ter sofrido viés de informação, uma vez que alguns indivíduos não reconheciam a unidade de saúde que era responsável pelo seu domicílio. Além disso, a categorização da variável cobertura da atenção primária à saúde em áreas não cobertas pela ESF pode ter influenciado as frequências de filas e dias específicos para marcação de consultas, haja vista que, nas unidades que priorizam o pronto atendimento, essas categorias apresentariam menores valores quando comparados aos das UBS tradicionais. Para tanto, foi implementado pelos pesquisadores um grande esforço na correção e adequação das respostas para essa variável, destacando que foram excluídos do estudo aqueles indivíduos que afirmaram ter procurado o serviço de atenção primária à saúde referência do seu domicílio, mas que preencheram o campo da unidade de saúde com o nome de outro serviço da rede pública do município.

Por outro lado, destaca-se como potencialidade do estudo a construção de um modelo teórico com base na literatura sobre o tema que guiou a seleção do elenco de variáveis e dos procedimentos de análise, além de permitir a interpretação dos achados. Aponta-se, ainda, que a realização de um inquérito domiciliar configura uma metodologia adequada para investigar acesso aos serviços de saúde. Enfatiza-se também o rigor metodológico na adequação dos métodos utilizados na análise estatística, haja vista que optou-se por uma estratégia metodológica que não vem sendo adotada rotineiramente em Epidemiologia, que é a estimação da RP por meio do modelo logístico multinível. Desse modo, para responder de maneira inequívoca à pergunta científica central do estudo, foram utilizadas estratégias alternativas (modelo Poisson multinível) cujos resultados permitem não somente avaliar a robustez da análise escolhida, como tornar possível a comparação com resultados de outros estudos que não adotem o uso da RP, mas sim da OR (estimada também pelo modelo logístico multinível). Além disso, como há um extenso debate na literatura epidemiológica sobre uso de OR versus RP, como também sobre os métodos de análise apropriados, então é essencial fundamentar a escolha metodológica nesta situação particular 30,31,46. É importante ainda ressaltar que a maior parte da discussão na literatura epidemiológica não é feita no contexto de modelos multiníveis utilizados para a correção de amostragem de conglomerados, o que torna ainda mais essencial a comparação dessas estimativas para permitir a compreensão do papel dessas estratégias de análise na estimação da RP em situações em que os dados são correlacionados.

Esta pesquisa evidenciou que os adolescentes e adultos jovens conseguem acessar os serviços de atenção primária à saúde, apesar da existência de barreiras de acesso geográficas e organizacionais. Isso indica que, embora seja considerada porta de entrada privilegiada e alternativa substitutiva do modelo biomédico, a atenção primária à saúde ainda enfrenta muitos desafios para a efetivação de um papel organizador e coordenador do cuidado no sistema de saúde.

Recomendam-se, portanto, novas investigações sobre o tema entre adolescentes e jovens, com a utilização de outras metodologias, como pesquisas qualitativas, que explorem com mais detalhes as barreiras que obstaculizam o acesso aos serviços primários de saúde. Além disso, faz-se necessário fortalecer políticas públicas já existentes com estratégia para inserir esse público no âmbito da atenção primária à saúde, ou seja, desenvolver mecanismos e repensar as práticas das equipes de saúde de forma a preconizar uma atenção acolhedora e de qualidade. O investimento em ações de prevenção e promoção da saúde na juventude pode contribuir para melhores condições de vida e saúde na idade adulta. 


\section{Colaboradores}

M. M. F. Martins contribuiu com a concepção e o projeto, análise e interpretação dos dados, redação do artigo e aprovação final da versão a ser publicada. R. Aquino colaborou com a concepção e o projeto, interpretação dos dados, revisão crítica relevante do conteúdo intelectual e aprovação final da versão a ser publicada. M. L. Pamponet participou da concepção do projeto, interpretação dos dados e revisão crítica relevante do conteúdo intelectual. E. P. Pinto Junior contribuiu com a interpretação dos dados, revisão crítica relevante do conteúdo intelectual e aprovação final da versão a ser publicada. L. D. A. F. Amorim colaborou com a análise e interpretação dos dados, revisão crítica relevante do conteúdo intelectual e aprovação final da versão a ser publicada.

\section{Informações adicionais}

ORCID: Maísa Mônica Flores Martins (0000-00018329-614X); Rosana Aquino (0000-0003-39065170); Marina Luna Pamponet (0000-0003-39067803); Elzo Pereira Pinto Junior (0000-0002-69772071); Leila Denise Alves Ferreira Amorim (00000002-1112-2332).

\section{Agradecimentos}

Ao Conselho Nacional de Desenvolvimento Científico e Tecnológico (CNPq) pela concessão das bolsas de estudo para pós-graduação (mestrado e doutorado).

\section{Referências}

1. Ruzany MH. Atenção à saúde do adolescente: mudança de paradigma. In: Departamento de Ações Programáticas Estratégicas, Secretaria de Atenção à Saúde, Ministério da Saúde, organizador. Saúde do adolescente: competências e habilidades. Brasília: Editora do Ministério da Saúde; 2008. p. 21-5. (Série B. Textos Básicos de Saúde).

2. Furtado EZL, Gomes KRO, Gama SGN. Acesso à assistência ao parto de adolescentes e jovens na região Nordeste do Brasil. Rev Saúde Pública 2016; 50:23.

3. Stopa SR, Malta DC, Monteiro CN, Szwarcwald CL, Goldbaum M, Cesar CLG. Acesso e uso de serviços de saúde pela população brasileira, Pesquisa Nacional de Saúde 2013. Rev Saúde Pública 2017; 51 Suppl 1:3s.

4. Claro LBL, March C, Mascarenhas MTM, Castro IAB, Rosa MLG. Adolescentes e suas relações com serviços de saúde: estudo transversal em escolares de Niterói, Rio de Janeiro, Brasil. Cad Saúde Pública 2006; 22:1565-74.

5. Dias-da-Costa JS, Gigante DP, Horta BL, Barros FC, Victora CG. Utilização dos serviços de saúde por adultos da coorte de nascimentos de 1982 a 2004-5, Pelotas, RS. Rev Saúde Pública 2008; 42 Suppl 2:51-9.

6. World Health Organization. Salud de los adolescentes. http://www.who.int/topics/adoles cent_health/es/ (acessado em 19/Mai/2016).

7. Braz M. Doenças crônicas e problemas de saúde em adolescentes do Município de Campinas [Dissertação de Mestrado]. Campinas: Faculdade de Ciências Médicas, Universidade Estadual de Campinas; 2011.

8. Klein JD, McNulty M, Flatau C. Adolescents' access to care: teenagers' self-reported use of services and perceived access to confidential care. Arch Pediatr Adolesc Med 1998; 152:676-82.

9. Kang M, Bernard D, Booth M, Quine S, Alperstein G, Usherwood T, et al. Access to primary health care for Australian young people: service provider perspectives. Br J Gen Pract 2003; 53:947-52.

10. Donabedian A. Los espacios de la salud: Aspectos fundamentales de la organización de la atención médica. Ciudad de México: Fondo de Cultura Económica; 1988.

11. Aday LA, Andersen R. A framework for the study of access to medical care. Health Serv Res 1974; 9:208-20.

12. Andersen RM. Reviting the behavioral model and access to medical care: does it matter? J Health Soc Behav 1995; 36:1-10.

13. Starfield B. Atenção primária: equilíbrio entre necessidades de saúde, serviços e tecnologia. Brasília: Organização das Nações Unidas para a Educação, a Ciência e a Cultura/Ministério da Saúde; 2002. 
14. Oliveira MM, Andrade SSCA, Campos MO, Malta DC. Fatores associados à procura de serviços de saúde entre escolares brasileiros: uma análise da Pesquisa Nacional de Saúde do Escolar (PeNSE), 2012. Cad Saúde Pública 2015; 31:1603-14.

15. Copland RJ, Denny SJ, Robinson EM, Crengle S, Ameratunga S, Dixon R. Self-reported pregnancy and access to primary health care among sexually experienced New Zealand high school students. J Adolesc Health 2011; 49:518-24.

16. Booth ML, Bernard D, Quine S, Kang MS, Usherwood T, Alperstein G, et al. Access to health care among Australian adolescent's young people's perspectives and their sociodemographic distribution. J Adolesc Health 2004; 34:97-103.

17. Palazzo LS, Béria JU, Tomasi E. Adolescentes que utilizan servicios de atención primaria: ¿Cómo viven? ¿Por qué buscan ayuda y cómo se expresan? Cad Saúde Pública 2003; 19:1655-65.

18. Muza GM, Costa MP. Elementos para a elaboração de um projeto de promoção à saúde e desenvolvimento dos adolescentes - o olhar dos adolescentes. Cad Saúde Pública 2002; 18:321-8.

19. Carvalho IE, Mello MB, Morais SS, Silva JLP. Fatores associados ao acesso anterior à gestação a serviços de saúde por adolescentes gestantes. Rev Saúde Pública 2008; 42:886-94.

20. Vieira RP, Gomes SHP, Machado MFAS, Bezerra IMP, Machado CA. Participação de adolescentes na Estratégia Saúde da Família a partir da estrutura teórico-metodológica de uma participação habilitadora. Rev Latinoam Enferm 2014; 22:309-16.

21. Rocha RACP, Goes PSA. Comparação do acesso aos serviços de saúde bucal em áreas cobertas e não cobertas pela Estratégia Saúde da Família em Campina Grande, Paraíba, Brasil. Cad Saúde Pública 2008; 24:2871-80.

22. Quinderé PHD, Jorge MSB, Nogueira MSL, Costa LFA, Vasconcelos MGF. Acessibilidade e resolubilidade da assistência em saúde mental: a experiência do apoio matricial. Ciênc Saúde Coletiva 2013; 18:2157-66.

23. Silva ZP, Ribeiro MCSA, Barata RB, Almeida MF. Perfil sociodemográfico e padrão de utilização dos serviços de saúde do Sistema Único de Saúde (SUS), 2003-2008. Ciênc Saúde Coletiva $2011 ; 16: 3807-16$

24. Aquino R. Avaliação dos efeitos da Estratégia Saúde da Família: adoção de hábitos saudáveis e acessibilidade a serviços primários de promoção da saúde e prevenção de riscos e agravos. Relatório final do projeto. Salvador: Instituto de Saúde Coletiva, Universidade Federal da Bahia; 2012.
25. Departamento de Planejamento, Monitoramento e Avaliação em Saúde, Prefeitura Municipal de Camaçari. Plano municipal de saúde 2014-2017. Camaçari: Prefeitura Municipal de Camaçari; 2014.

26. Ramos DD, Lima ADS. Acesso e acolhimento aos usuários em uma unidade de saúde de Porto Alegre, Rio Grande do Sul, Brasil. Cad Saúde Pública 2003; 19:27-34.

27. Souza MSPL, Aquino R, Pereira SM, Costa MCN, Barreto ML, Natividade M, et al. Fatores associados ao acesso geográfico aos serviços de saúde por pessoas com tuberculose em três capitais do Nordeste brasileiro. Cad Saúde Pública 2015; 31:111-20.

28. Rao JNK, Scott AJ. The analysis of categorical data from complex sample surveys: chisquared tests for goodness of fit and independence in two-way tables. J Am Stat Assoc 1981; 76:221-30.

29. Rao JNK, Scott AJ. On chi-squared tests for multiway contingency tables with cell proportions estimated from survey data. Ann Stat 1984; 12:46-60.

30. Santos CAST, Fiaccone RL, Oliveira NF, Cunha S, Barreto ML, Carmo MB, et al. Estimating adjusted prevalence ratio in clustered cross-sectional epidemiological data. BMC Med Res Methodol 2008; 8:80.

31. Localio AR, Margolis DJ, Berlin JA. Relative risks and confidence intervals were easily computed indirectly from multivariable logistic regression. J Clin Epidemiol 2007; 60:874-82.

32. Instituto Brasileiro de Geografia e Estatística. Pesquisa Nacional de Saúde 2013. Acesso e utilização dos serviços de saúde, acidentes e violências: Brasil, grandes regiões e Unidades da Federação. Rio de Janeiro: Instituto Brasileiro de Geografia e Estatística; 2015.

33. Instituto Brasileiro de Geografia e Estatística. Pesquisa Nacional por Amostra de Domicílios. Um panorama da saúde no Brasil: acesso e utilização dos serviços, condições de saúde e fatores de risco e proteção à saúde 2008. Rio de Janeiro: Instituto Brasileiro de Geografia e Estatística; 2010.

34. Instituto Brasileiro de Geografia e Estatística. Pesquisa Nacional de Saúde do Escolar, 2012. Rio de Janeiro: Instituto Brasileiro de Geografia e Estatística; 2013.

35. Nunes BP, Flores TR, Duro SMS, Saes MO, Tomasi E, Santiago AD, et al. Utilização dos serviços de saúde por adolescentes: estudo transversal de base populacional, Pelotas-RS, 2012. Epidemiol Serv Saúde 2015; 24:411-20. 
36. Oliveira LS, Almeida LGN, Oliveira MAS, Gil GB, Cunha ABO, Medina MG, et al. Acessibilidade a atenção básica em um distrito sanitário de Salvador. Ciênc Saúde Coletiva 2012; 17:3047-56.

37. Olsen JM, Lago TDG, Kalckmann S, Alves MCGP, Escuder MML. Práticas contraceptivas de mulheres jovens: inquérito domiciliar no Município de São Paulo, Brasil. Cad Saúde Pública 2018; 34:e00019617.

38. Santos AAG, Silva RM, Machado MFAS, Vieira LJES, Catrib AMF, Jorge HMF. Sentidos atribuídos por profissionais à promoção da saúde do adolescente. Ciênc Saúde Coletiva 2012; 17:1275-84.

39. Ayres JRCM. Adolescência e saúde coletiva: aspectos epistemológicos da abordagem programática. In: Schraiber LB, organizadora. Programação em saúde hoje. 2a Ed. São Paulo: Editora Hucitec; 1993. p. 139-82.

40. Marques JF, Queiroz MVO. Cuidado ao adolescente na atenção básica: necessidades dos usuários e sua relação com o serviço. Rev Gaúch Enferm 2012; 33:65-72.

41. Battesini M, Coelho HS, Seta MH. Uso de programação linear para otimizar o acesso geográfico em redes temáticas de atenção à saúde. Cad Saúde Pública 2018; 34:e00055017.
42. Silva Júnior ES, Medina MG, Aquino R, Fonseca ACF, Vilasbôas ALQ. Acessibilidade geográfica à atenção primária à saúde em distrito sanitário do município de Salvador, Bahia. Rev Bras Saúde Matern Infant 2010; 10 Suppl 1:S49-60.

43. Lima SAV, Silva MRF, Carvalho EMF, Cesse EAP, Brito ESV, Braga JPR. Elementos que influenciam o acesso à atenção primária na perspectiva dos profissionais e dos usuários de uma rede de serviços de saúde do Recife. Physis (Rio J.) 2015; 25:635-56.

44. Cunha ABO, Vieira-da-Silva LM. Acessibilidade aos serviços de saúde em um município do Estado da Bahia, Brasil, em gestão plena do sistema. Cad Saúde Pública 2010; 26:725-37.

45. Hulka BS, Wheat JR. Patterns of utilization: the patient perspective. Med Care 1985; 23:43860.

46. Barros AJD, Hirakata VN. Alternatives for logistic regression in cross-sectional studies: an empirical comparison of models that directly estimate the prevalence ratio. BMC Med Res Methodol 2003; 3:21. 


\section{Abstract}

The study sought to analyze the association between adolescents and young adults' access to primary health care services and Family Health Strategy (FHS) services. It is a cross-sectional study based on a household survey of 812 individuals aged between 15 and 24 years in Camaçari, Bahia State, Brazil, through cluster sampling. Demographic, socioeconomic, health and health service variables were used to describe the population according to primary health care area. The association between primary health care coverage area and access to its services, access barriers and participation in educational activities were estimated through prevalence ratios $(P R)$ using multilevel logistic regression models in the $R$ software. Access to primary health care services was reported by $89.5 \%$ of individuals, with no statistically significant differences between this type of access and primary health care coverage area. However, among individuals who reside in areas covered by FHS, there was greater access to harm reduction and health promotion actions $(P R=3.0$; 95\% CI: 1.68-5.34), but also lower probability of being seen by a health worker on the same day the appointment was scheduled (PR $=0.60 ; 95 \% \mathrm{CI}$ : 0.48-0.74) and lower availability of public transportation ( $R P=0.59$; 95\%CI: 0.39-0.90). Study results point to a high prevalence of access to primary health care services among adolescents and young adults. Though we did not observe a difference in access between areas with FHS coverage, young people's greater participation in prevention and health promotion activities reinforces this strategy's importance within Brazilian Unified National Health System (SUS).

Health Services Accessibity; Primary Health Care; Family Health Strategy; Adolescent

\section{Resumen}

El objetivo de este estudio fue analizar la asociación entre el acceso a los servicios de atención primaria en salud de adolescentes y adultos jóvenes y la cobertura de la Estrategia de Salud Familiar (ESF). Se trata de un estudio transversal, a partir de una encuesta domiciliaria, realizada con 812 individuos de 15 a 24 años de edad, en Camaçari, Bahía, Brasil, mediante una muestra por conglomerados. Se utilizaron variables demográficas, socioeconómicas, de salud y de servicios de salud para la descripción de la población, según el área de cobertura de atención primaria en salud. La asociación entre área de cobertura de la atención primaria en salud y el acceso a los servicios de esta, las barreras de acceso, y la participación en actividades educativas se estimaron mediante la razón de prevalencia $(R P)$, con el uso del modelo de regresión logística multinivel en el software $R$. Un 89,5\% de los individuos informó de acceso a servicios de atención primaria en salud, no existiendo diferencias estadisticamente significativas entre este tipo de acceso y el área de cobertura de la ESF. No sólo entre individuos residentes en áreas cubiertas por la ESF se verificó un mayor acceso a las acciones de prevención de enfermedades y promoción de la salud ( $R P=3,0$; IC95\%: 1,68-5,34), sino también una menor probabilidad de que la atención se produzca el mismo día en el que se fija la consulta $(R P=0,60$; IC95\%: 0,48-0,74) y una menor disponibilidad de transporte colectivo $(R P=$ 0,59; IC95\%: 0,39-0,90). Los datos de este estudio apuntaron una elevada prevalencia de acceso a los servicios de la atención primaria en salud entre los adolescentes y adultos jóvenes. A pesar de que no se hayan observado diferencias de acceso entre las áreas con cobertura de la ESF, la mayor participación de estos individuos en actividades de prevención y promoción de la salud refuerza la importancia de esta estrategia en el ámbito del SUS.

Accesibilidad a los Servicios de Salud; Atención

Primaria de Salud; Estrategia de Salud

Familiar; Adolescente
Recebido em 07/Mar/2018

Versão final reapresentada em 31/Ago/2018

Aprovado em 24/Set/2018 